\title{
Clinical Observation of Three Dimensional Conformal Radiotherapy with Tamoxifen in Treatment of Postoperative Malignant Glioma
}

\author{
Shao-Bing Zhou ${ }^{1 \&}$, Yang-Chen Liu ${ }^{1,2 \&}$, Xiao-Xiang Yin', Wen-Xiu Ding ${ }^{1}$, Xin-Wei \\ Guo ${ }^{1}$, Liang Gu${ }^{1}$, Xin-En Huang ${ }^{2 *}$
}

\begin{abstract}
Objective: To evaluate the efficacy and adverse effects of three dimensional conformal radiotherapy (3D-CRT) with tamoxifen in treating patients with postoperative malignant glioma. Patients and Methods: 60 patients of postoperative malignant glioma were randomly assigned into two groups, 30 patients were treated with 3D-CRT plus tamoxifen (treatment group), and the other 30 patients with 3D-CRT plus temozolomide (control group). All patients were radiated by $6 \mathrm{MV}$ X-ray, $2.0 \mathrm{~Gy}$ per fraction, once daily, with a total dose (DT) of 56 60Gy. Tamoxifen was delivered at $60 \mathrm{mg} / \mathrm{m}^{2} / \mathrm{d}$, temozolomide was given at $75 \mathrm{mg} / \mathrm{m}^{2} / \mathrm{d}$. All patients were treated with concurrent radiotherapy. Results: One, 2, 3 year survival rates of treatment and control group were $63.3 \%$, $30.0 \%, 23.0 \%$ and $70.0 \%, 33.3 \%, 26.7 \%$, respectively $\left(\chi^{2}=0.01,0.23,0.09, P>0.05\right)$. The rate of thromboembolism in treatment group was $6.7 \%$. Conclusion: Therapeutic efficacy of two groups was similar, but it was more costeffective in treatment group, and toxicity did not increase.
\end{abstract}

Keywords: Malignant glioma - three dimensional conformal radiotherapy - tamoxifen

Asian Pac J Cancer Prev, 16 (5), 1743-1745

\section{Introduction}

Malignant glioma is one the most common brain tumor, and surgery is the principal treatment method. Because of its invasive growth, surgery is difficult to eradicate glioma, thus, postoperative concomitant chemoradiotherapy is considered to be a routine treatment (Yin et al ., 2008; Pashaki et al., 2014). However, the treatment effect is not encouraging. To explore new treatment, we conducted a clinical investigation from June 2006 to July 2011, by using three dimensional conformal radiotherapy (3D-CRT) in combination with tamoxifen (treatment group) or 3D-CRT combined with temozolomide (control group) respectively in the treatment of brain malignant glioma. The details are reported as follows.

\section{Materials and Methods}

\section{Patient}

Eligible 60 patients who meet the following conditions: (1) most or partial of the tumors were removed by surgery; (2) Grade III or IV of astrocytoma or glioblastoma confirmed by postoperative histological pathology. (3) The Karnofsky score no less than 60 points, and life expectancy>3 months; (4) no obvious contraindication for treatment, agreed to enrolled the group, and signed up the informed consent; (5) did not receive endocrine therapy such as tamoxifen and so on, or radiochemotherapy before. 34 were male and 26 female, age between 14 and 75 years, with a median age 45.7 years old. Randomly divided to 3D-CRT in combination with tamoxifen (treatment group) and 3D-CRT combined with temozolomide (control group), 30 patients in each group. Clinical characteristics in two groups were comparable $(p>0.05)$, for details see Table 1.

\section{Treatment}

In 2 5 weeks after surgery, every patient was examined by enhanced MRI imaging to estimate tumor target in head. We use the head and neck shoulder fixator and the thermoplastic mask to fix the patient's body, then take spiral enhanced CT scan for head, $2 \mathrm{~mm}$ thick, and then CT images transferred via the network to TPS. After image reconstruction, on the basis of head MRI and CT positioning, three or more doctors delineated the target volume and the organs at risk before radiotherapy. GTV (gross tumor volume) include tumors and edema area, CTV created by giving a $2.5 \mathrm{~cm}$ margin to GTV, and PTV was CTV plus $0.5 \mathrm{~cm}$, using 3 5 radiation field. PTV covered more than $90 \%$ isodose line, using $6 \mathrm{MV}$ X-ray produced by linear accelerator named Presise, manufactured by ELEKTA medical company. The prescribed total dose was

${ }^{1}$ Department of Radiation Oncology, Affiliated People's Hospital of Yangzhou University, Taixing, ${ }^{2}$ Department of Chemotherapy, the Affiliated Jiangsu Cancer Hospital of Nanjing Medical University and Jiangsu Institute of Cancer Research, Nanjing, China ${ }^{\star}$ Equal contributors *For correspondence: huangxinen06@163.com 
Table 1. Clinical characteristics of patients in two groups (n)

\begin{tabular}{lccc}
\hline Group & Treatment Group* Control Group* & $p$ \\
\hline $\begin{array}{l}\text { Total namber } \\
\text { of cases }\end{array}$ & 30 & 30 & \\
Gender & & & \\
$\quad$ Male & 18 & 16 & 0.100 \\
Female & 12 & 14 & \\
Age & 45.5 & 45.9 & 0.056 \\
KPS score & $73.4 \pm 12.3$ & $73.6 \pm 12.5$ & 0.08 \\
Operation & & & \\
R0 & 6 & 5 & \\
R1 & 17 & 19 & 0.780 \\
R2 & 7 & 6 & \\
Pathological type & & & \\
Grade III gliomas & 18 & 19 & \\
Grade IV gliomas & 8 & 8 & 0.852 \\
Glioblastoma & 4 & 3 & \\
\hline
\end{tabular}

*Treatment group, 3D-CRT in combination with tamoxifen; control group, 3D-CRT combined with temozolomide

56 60 Gy with conventional fraction, 2.0 Gy per fraction, one fraction per day, treated from Monday through Friday. Tamoxifen was given orally $60 \mathrm{mg} / \mathrm{m}^{2}, 3$ times a day in the treatment group, and Temozolomide $75 \mathrm{mg} / \mathrm{m}^{2}$, once a day in the control group, and both were taken continuously from the first day to the end of radiotherapy.

\section{Response and toxicity}

CT or MRI was reviewed every 3 months for the first year after treatment, every six months for the second and the third years, and the treatment effect was evaluated according to WHO standards (response evaluation criteria in solid tumor-RECIST)respectively, was divided into: CR, PR, SD and PD. The acute toxicities and late complications were assessed by the RTOG standard.

\section{Statistical method}

We used SPSS (version17.0 Inc. Chicago, IL, USA) for statistical analyses. For all patients, median survival was estimated by Kaplan-Meier method. Effect on survival of any variable was estimated according to log-rank test. $P$ value less than 0.05 was considered as significant.

\section{Results}

Every patient in two groups completed the treatment plan, and was reviewed after treatment for 3 years. The follow-up rate was $100 \%$. Response to treatment was recorded as follow: in treatment group, $5 \mathrm{CR}, 14 \mathrm{PR}, 5$ SD and 6 PD; and in control group, 15 PR, 3 SD and 5 PD. The response rate of each group was $63.3 \%$ and $73.3 \%\left(\chi^{2}=0.69, p>0.05\right)$. One, 2,3 year survival rates of treatment and control group were $63.3 \%, 30.0 \%, 23.0 \%$ and $70.0 \%, 33.3 \%, 26.7 \%$, respectively (chi-square $=0.01$, $0.23,0.09, p>0.05$ ) (Figure 1), and the median survival was 16.1 months and 18.2 months, respectively.

Toxicity included cerebral edema, with the incidence of $30.0 \%$ in treatment and $33.3 \%$ in control group, all of recovered after supportive treatment. Bone marrow depression for more than Grade2 was $16.7 \%$ and $23.3 \%$ respectively. The incidence of thromboembolism in the treatment group was $6.7 \%$. Hair loss was at a similar incidence in the two groups. Long-term toxicities were mainly memory loss and mental injury, the incidence was $40 \%$ and $50 \%$ in treatment and control group respectively. No radioactive brain necrosis was recorded.

\section{Discussion}

Glioma is one of the most common tumors of the central nervous system, accounted for $45 \%$ 50\% of tumors in central nervous system. Surgery is still the principal treatment choice for patients with glioma, but due to the anatomy of the central nervous system and the characteristics of the invasive tumor growth, surgery is difficult to eradicate the lump in most cases, and postoperative radiotherapy is considered a routine treatment (Tang et al., 2009; Zheng et al., 2011). Due to low sensitivity to radioactive of the glioma cells, and conventional radiotherapy dose increased to the tumor area could destroy normal tissue seriously. 3D-CRT or intensity-modulated radiotherapy (IMRT) could concentrate the target dose precisely, and further reduce the dose to normal tissues and organs. Therefore could improve the local control ratio, and reduce irradiation injury to normal tissue. However, if the growth of the malignant tumor could be controled is not clear (Wang et al., 2011).

For malignant glioma, surgery, radiotherapy and chemotherapy is considered to improve the median survival time of patients for less than one year, and the effect of surgery combined with radiotherapy has reached a plateau. Chemotherapy has a potential to improve the survival time, but still reported less effective. Temozolomide is reported to get through the blood-brain barrier, and has synergistic effect with radiotherapy, therefore become a standard treatment for malignant glioma after surgery. But the effect has barely increased, and with the following deficiencies: (1) increased side effects to the central nervous system and other systems. (2) tumor cells are prone to resistance to chemotherapy, lead to treatment effect of chemotherapy less significant (Partap S et al., 2007; wang et al., 2013); (3) the expensive medical cost of temozolomide limits its application. To find a medication with cheap price, higher efficiency and lower toxicity are is our aim. Researches showed that proliferation of high grade glioma relies partly on the activation of protein kinase C (PKC) pathway, thus blocking this enzyme could inhibit the growth of glioma cells. Tamoxifen, an anti-estrogen drug, is a very important PKC inhibitor, could inhibit the activity of PKC glioma cells and protein expression, thereby inhibiting glioma cell proliferation and induce apoptosis. Tamoxifen is reported to get through the blood-brain barrier, and with lower cost and toxicities, as well as with a radiosensitization effect on radiotherapy (Pollack et al., 1997; Zhang et al., 2000 ). Broniscer etc. reported that high dose of tamoxifen administered with or after conventional radiotherapy could inhibit PKC, and improve the curative effect and tolerance when treating patients with malignant glioma. A previous clinical study suggested that tamoxifen could play an important role in the treatment of high grade glioma, no matter in the initial therapy or during 
Three Dimensional Conformal Radiotherapy with Tamoxifen for Postoperative Treatment of Malignant Glioma

combined radiotherapy and chemotherapy (Flannery et al., 2005). The maximum tolerated dose of tamoxifen used in combination treatment is $100 \mathrm{mg} / \mathrm{m}^{2}$. Recent research also suggests that tamoxifen could also play as a protectant to alleviate nerve inflammation reaction process, which induced by small and medium-sized glial cells, and thus with a protection effect on normal nerve cells (Tian et al., 2009).

On this background, we studied postoperative patients with malignant glioma, using 3D-CRT in combination with tamoxifen (treatment group) and 3D-CRT combined with temozolomide (control group), and the 1, 2, 3 years survival rates were $63.3 \%, 30.0 \%, 23.0 \%$ and $70.0 \%$, $33.3 \%, 26.7 \%$ respectively $\left(\chi^{2}=0.01,0.23,0.09, P>0.05\right)$. This result is slightly higher than what Robins, etc. reported (Robins et al., 2006). In their report they also combined with tamoxifen group (Robins et al., 2006). and the reason maybe as follow: (1) the 3D-CRT midway is not wild, improved the original site around dose, and so increased the local control and survival rate. (2) tamoxifen was delivered for $60 \mathrm{mg} / \mathrm{m}^{2}, 3$ times a day, continued for two months, rather than $80 \mathrm{mg} / \mathrm{m}^{2}, 4$ times a day, until disease progression, thereby reducing the side effects, and with a survival benefit. But lower than combined temozolomide Group reported by Liu Xing-xiang, etc., maybe the reason is as follows: (1) in this research (Liu et al., 2009), temozolomide was no longer doses at the end of radiotherapy; (2) in our research, some patients were Grade IV astrocytoma, while in Liu Xing-xiang's report, patients were only grade III. In this study, the incidence of thrombosis was $6.7 \%$, significantly lower than Robins reported $20.8 \%$ (Robins et al., 2006).

In conclusion, we suggested that therapeutic efficacy of two groups was similar, but it was more cost-effective in tamoxifen group, and toxicity did not increase.

\section{Acknowledgements}

Dr. Xin-En Huang is supported in part by the project of science and technology of Nanjing science committee. (Grant 201303046) and Jiangsu Provincial Special Program of Medical Science (BL2014092).

\section{References}

Broniscer A, Leite CC, Lanchote, et al (2000). Radiation therapy and high-dose tamoxifen in the treatment of patients with diffuse brainstem gliomas: results of a Brazilian cooperative study. J Clin Oncol, 18, 1246-53.

Flannery TW, Dibiase S, Patel S, et al (2005). A Phase I clinical trial assessing temozolomide and tamoxifen with concomitant radiotherapy for the treatment high-grade gliomas. Int J Radiat Oncol Biol Phys, 82, 739-42.

Liu XX, Cui L, Dong HM (2009). Clinical observation in 3 dimensional conformal radiotherapy (3D-CRT) concurrent chemotherapy in treatment of postoperative cerebral gliomas. China Oncology, 12, 938-42.

Partap S, Fisher PC (2007). Update on new treatments and developments in childhood brain tumors. Curt Opin Pediart, 19, 670-4.

Pashaki AS, Hamed EA, Mohamadian K, et al (2014). Efficacy of high dose radiotherapy in post-operative treatment of glioblastoma multiform--a single institution report. Asian Pac J Cancer Prev, 15, 2793-6.

Pollack IF, DaRosso RC, Robertson PL, et al (1997). A phase I study of high-dose tamoxifen for the treatment of refractory malignant gliomas of childhood. Clin Cancer Res, 3, 110915.

Robins H, Won M, Seiferheld WF, et al (2006 ). Phase 2 trial of radiation plus high-dose tamoxifen for glioblastoma multiforme. Neuro oncolo, 8, 47-52.

Tang Xi, Hu Ya, Xu Yanhua (2009). Clinical research in three-dimensional conformal radiotherapy combined with Temozolomide in the treatment of malignant glioma. Prog Modern Biomedicine, 12, 2885-6.

Tian DS, Liu JL, Xie MJ, et al (2009). Tamoxifen attenuates inflammatory-mediated damage and improves functional outcome after spinal cord injury in rats. J Neurochem, 109, 1658-67.

Wang JJ, Wang G, Du SM, et al (2013). Relations between multidrug resistance and P13K/Akt signaling pathway and treatment progression of glioma. Oncology Progress, 11, 287-91.

Wang LY, Tu QS, Zhou WB, et al (2011). Efficacy and safety of 3-dimensional conformal radiotherapy combined with Temozolomide for glioma. J Central South University, 4, 1106-10.

Yin WB, Yu ZH, Xu GZ, et al (2008). Beijing: Beijing union medical university press, Radiation Oncology, 1122-9.

Zhang W, Couldwell WT, Song H, et al (2000). Tamoxifeninduced enhancement of calcium signaling in glioma and MCF-7 breast cancer cells. Cancer Res, 60, 395-400.

Zheng W, Nie Q, Kang JB, et al (2011). Clinical efficacy study of concurrent chemoradiotherapy for patients with postoperative high grade glioma. Chinese J Neuromedicine, 10, 900-4. 